\title{
BMJ Open Cardiovascular morbidity and mortality in patients with type 2 diabetes using novel antidiabetic medicines as add-on therapy: an observational real- world study
}

\author{
Spela Zerovnik (D), Mitja Kos (D), Igor Locatelli (D)
}

To cite: Zerovnik S, Kos M, Locatelli I. Cardiovascular morbidity and mortality in patients with type 2 diabetes using novel antidiabetic medicines as add-on therapy: an observational real-world study. BMJ Open 2021;11:e051549. doi:10.1136/ bmjopen-2021-051549

- Prepublication history and additional supplemental material for this paper are available online. To view these files, please visit the journal online (http://dx.doi.org/10.1136/ bmjopen-2021-051549).

Received 22 March 2021 Accepted 24 August 2021

Check for updates

(C) Author(s) (or their employer(s)) 2021. Re-use permitted under CC BY-NC. No commercial re-use. See rights and permissions. Published by BMJ.

University of Ljubljana, Faculty of Pharmacy, Ljubljana, Slovenia

Correspondence to

Dr Igor Locatelli;

igor.locatelli@ffa.uni-lj.si

\section{ABSTRACT}

Objective To evaluate the effect of sodium-glucose co-transporter 2 inhibitors (SGLT2i) and glucagon-like peptide-1 receptor agonists (GLP-1RA), compared with dipeptidyl peptidase-4 inhibitors (DPP-4i) as add-on therapy on cardiovascular (CV) morbidity and mortality in patients with type 2 diabetes (T2D).

Design and setting $A$ nationwide cohort study using three linked healthcare databases from Slovenia (outpatient prescription claims data, hospitalisation claims data and death registry data).

Participants Patients with T2D with newly introduced DPP-4i $(n=3817)$, GLP-1RA $(n=855)$ or SGLT2i $(n=2851)$ add-on therapy between June 2014 and June 2018. Primary and secondary outcome measures The primary outcome was a major adverse CV event (MACE), while the secondary outcomes were CV death and heart failure (HF). The effects of the antidiabetic medicine group on the risk of each outcome were estimated with Cox proportional hazards regression. Intention-to-treat and on-treatment approaches were used.

Results In the intention-to-treat analysis, SGLT2i as add-on therapy, when compared with DPP-4i, was associated with lower risk of MACE (HR $=0.66 ; 95 \% \mathrm{Cl}$ 0.50 to $0.85 ; \mathrm{p}=0.002)$ and $\mathrm{CV}$ death $(\mathrm{HR}=0.46 ; 95 \% \mathrm{Cl}$ 0.30 to $0.73 ; p=0.001)$. On-treatment analysis revealed lower $\mathrm{HF}$ risk in patients initiating SGLT2i $(\mathrm{HR}=0.54$; $95 \% \mathrm{Cl} 0.30$ to $0.99 ; \mathrm{p}=0.047)$. In the intention-to-treat analysis, GLP-1RA add-on therapy was associated with a lower MACE risk when compared with DPP-4i ( $\mathrm{HR}=0.64 ; 95 \% \mathrm{Cl} 0.43$ to $0.97 ; \mathrm{p}=0.034$ ), but it had a non-significant effect on $\mathrm{CV}$ death $(\mathrm{HR}=0.62 ; 95 \% \mathrm{Cl}$ 0.34 to $1.14 ; p=0.128)$ and $\mathrm{HF}(\mathrm{HR}=1.39 ; 95 \% \mathrm{Cl}$ 0.88 to $2.21 ; p=0.157)$. The results of on-treatment analyses were in agreement with the results of intention-to-treat analyses.

Conclusions SGLT2i and GLP-1RA improved CV morbidity and mortality in patients with T2D when compared with DPP-4i as an add-on therapy. The results of this study may serve as a basis for the selection of an optimal add-on antidiabetic medicine to reduce $\mathrm{CV}$ morbidity and mortality in patients with $\mathrm{T} 2 \mathrm{D}$ in clinical practice.

Trial registration number EUPAS32558.

\section{STRENGTHS AND LIMITATIONS OF THIS STUDY}

$\Rightarrow$ This was the first study that directly compared cardiovascular outcomes and mortality of patients starting dipeptidyl peptidase-4 inhibitors, sodiumglucose co-transporter 2 inhibitors and glucagonlike peptide-1 receptor agonists as add-on therapy.

$\Rightarrow$ The relatively long follow-up period in this study enabled us to detect differences in cardiovascular events and death that may not be apparent at the shorter follow-up durations.

$\Rightarrow$ Cox proportional regression models included all available confounding variables that might influence the effect of antidiabetic medicine group on the outcome of interest.

$\Rightarrow$ The results of the on-treatment analyses and other sensitivity analyses were consistent with the results of the primary analyses (intention-to-treat analyses).

$\Rightarrow$ We were not able to control for some confounding variables (eg, patient body mass index, glycaemic control, cardiovascular disease control) because these data were not available in the databases used in this study.

\section{INTRODUCTION}

Randomised placebo-controlled trials reveal that treatment with sodium-glucose co-transporter 2 inhibitors (SGLT2i) and glucagonlike peptide-1 receptor agonists (GLP-1RA) reduces the risk of cardiovascular (CV) events in patients with type 2 diabetes (T2D). Specifically, GLP-1RA and SGLT2i reduce the risk of major adverse CV events (MACE), while SGLT2i also reduce the risk of heart failure (HF). Moreover, empagliflozin and liraglutide also reduce the risk of $\mathrm{CV}$ death, while other SGLT2i and GLP-1RA do not. ${ }^{1-8}$ Several observational studies have evaluated the effect of SGLT2i and GLP-1RA on the $\mathrm{CV}$ outcomes of patients with T2D in routine clinical practice. The majority of these studies have been based on electronic health records 
and/or registries from either the $\mathrm{USA}^{9-13}$ or Scandinavia. ${ }^{14-17}$ Studies that assess HF risk following SGLT2i treatment ${ }^{9-1214-1618-20}$ provide evidence that SGLT2i have a protective effect on HF, irrespective of the comparator used. In contrast, studies that compare the effects of dipeptidyl peptidase-4 inhibitors (DPP-4i) and GLP1RA on HF provide conflicting evidence. ${ }^{131721}$ Current evidence of how SGLT2i and GLP-1RA treatment affects the risk of MACE and mortality is inconclusive. ${ }^{14-161921}$ In contrast to the reduction of HF risk that appears shortly after the introduction of SGLT2i, ${ }^{1}{ }^{18}$ the detection of any changes to the risk of MACE or mortality likely require longer follow-up. Although most previous studies followed the outcomes of large groups of patients with T2D, many failed to use a specific study comparator ${ }^{11} 1419$ or were subject to an immortal time bias. ${ }^{22}$ In addition, the majority of previous SGLT2i studies that did use a specific comparator (mostly DPP-4i) had a relatively short follow-up period, usually less than 1 year in the primary analysis. ${ }^{9} 1012151618$ Few studies distinguish between the additional administration of a novel antidiabetic medicine alongside an existing therapy ('add-on therapy') and switching from background antidiabetic treatment to a novel antidiabetic ('switch'). Current guidelines ${ }^{23}$ recommend stepwise addition of antidiabetic medicines for patients who do not achieve glycaemic targets. Therefore, the aim of the current study was to evaluate the effect of SGLT2i and GLP-1RA, compared with DPP-4i as add-on therapy, on CV morbidity and mortality in real-world patients with T2D.

\section{METHODS}

This nationwide retrospective cohort study was conducted using data from three Slovenian databases (Outpatient Prescription Medicines Database, National Hospital Health Care Statistics Database, Causes of Death Registry) obtained from the National Institute of Public Health. The data were obtained for the period 1 January 2009 to 31 December 2019. The databases used in the study contain data on all dispensed outpatient prescriptions, all hospital admissions and deaths for the entire Slovenian population and are linkable at the patient level through anonymous patient identifier. A detailed description of the databases is included in the online supplemental material. The study protocol was registered at ENCePP.

\section{Cohort selection}

Figure 1 presents a flow chart of cohort selection. Database population was defined from outpatient prescriptions database. All patients who filled at least two prescriptions for any antidiabetic medicine within the 1-year period prior to 30 June 2014 (the date when the first SGLT2i became available on the Slovenian market) were included in the database population. For these patients, data on hospital admissions (from 1 January 2009 to 31 December 2019) and data on deaths were included in the database. We further excluded patients aged 40 years or less (to reduce the number of patients with T1D, gestational diabetes or early-onset T2D in our cohort) and patients previously treated with novel antidiabetic medicines (DPP-4i, GLP-1RA or SGLT2i; to ensure more homogeneous and comparable groups, as these patients could not be previously treated with SGLT2i but could be treated with GLP-1RA or DPP-4i).

From this patient population, we selected patients who received a first prescription for a novel antidiabetic medicine (see online supplemental table S1) between 30 June 2014 and 30 June 2018 ('incident-user design'). We further excluded patients who did not receive an additional prescription for a novel antidiabetic medicine (too short exposure time) and patients who received a comparator medicine within 135 days after index date (to isolate the effect of individual novel antidiabetic medicine group). Only patients with novel antidiabetic medicine add-on therapy were included in the final cohort (study population). To be considered as a patient with add-on therapy, patient had to refill a prescription for a novel antidiabetic medicine group within 135 days after index date (date of first filled prescription for newly introduced drug) and remain on continuous treatment with all medicines from his background antidiabetic therapy throughout 135 days after index date. If a patient did not remain on continuous treatment with his background antidiabetic therapy, this patient was deemed a switcher. A 135-day period was chosen to define add-on therapy as prescriptions for antidiabetic medicines in Slovenia are usually issued for a 90-day supply, to which a 45-day gap period was added. Patients were further divided in three subgroups according to which antidiabetic medicine group they were prescribed on index date. Follow-up started 135 days after index date ('start date'). Baseline characteristics of patients were evaluated on the start date; definitions of baseline patient characteristics are presented in online supplemental table S2.

\section{Outcomes}

ICD-10-AM (International Classification of Diseases, 10th Revision, Australian Modification, Sixth Edition) codes were used to define outcomes. We evaluated four outcomes: (1) MACE, (2) CV death, (3) HF and (4) allcause death. MACE was defined as main diagnosis of myocardial infarction (codes: I21, I22), ischaemic stroke (codes: I63, I64) or CV death, specified as primary cause of death with codes I00-I99. ${ }^{151624} \mathrm{HF}$ was defined as a main diagnosis of hospitalisation due to HF (code: I50). ${ }^{16} 18$

\section{Statistical analysis}

We evaluated the time from the start date to the date of the first event of interest for each antidiabetic medicine group using several Cox proportional regression models. We used an intention-to-treat (ITT) approach in the primary analysis. Patients were followed from the start date (Day 135 after index date) to the date of first event of interest, and they were censored on the date of death (last day of the month in which they died) or the end 


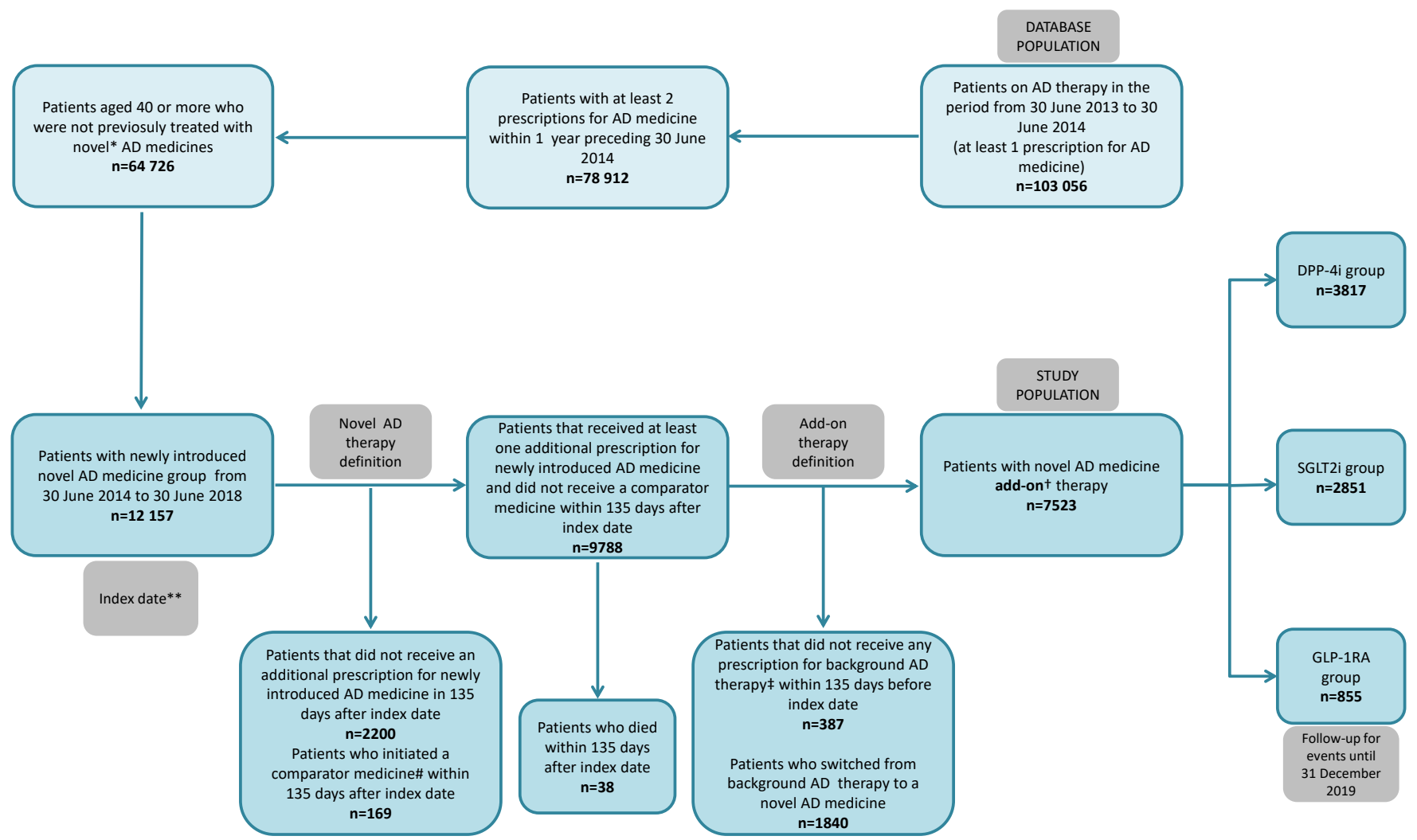

Figure 1 Flowchart of cohort selection. *novel antidiabetic medicines: DPP-4i, dipeptidyl peptidase-4 inhibitor; GLP-1RA, glucagon-like peptide-1 receptor agonist; SGLT2i, sodium-glucose co-transporter 2 inhibitor; **index date, date of a first prescription for a newly introduced antidiabetic medicine; \#comparator medicine, a medicine from another class of novel antidiabetic medicines; łexcluding insulin; †to be considered as a patient with add-on therapy, patient had to refill a prescription for a newly introduced medicine within 135 days after index date and remain on continuous treatment with all medicines from his background antidiabetic therapy throughout 135 days after index date. If a patient did not remain on continuous treatment with his background therapy, this patient was deemed a switcher. AD, antidiabetic.

of the study period (31 December 2019). Patients were followed irrespective of any changes to their antidiabetic therapy that occurred after the start date. The DPP-4i group was used as a reference category in all regression models. Additionally, we analysed associations between baseline patient characteristics and antidiabetic medicine group. For this purpose, we built several logistic regression models using dichotomous variables for patient characteristics as each dependent variable. For patient age, we used Analysis of variance (ANOVA).

We first developed a full covariate model for MACE outcome using an ITT approach (see online supplemental table S3), with all baseline patient characteristics as covariates (presented in table 1). We then developed final Cox regression models for all outcomes using an ITT approach. Due to small numbers of CV deaths and HF events, we reduced the number of used covariates; the number of events had to be at least eight times higher than the number of covariates (df) included in the models. ${ }^{25}$ For this purpose, we divided covariates in two blocks. The first block consisted of patient demographics (age, gender), proxies for well-controlled diabetes and diabetes severity (duration of diabetes therapy, use of insulin at baseline, hospitalisation due to T2D in the past year) and hospital admission due to different CV causes in the past year. These covariates were included in the model using Enter method. The second block consisted of covariates that describe various medicines in concomitant therapy, including other antidiabetic medicines in background therapy, such as metformin and sulphonylureas, and time of cohort entry. A stepwise backward selection, based on the Wald test (removal probability for stepwise selection was set to 0.2 ), was used for the second block of covariates. Additionally, we tested patient age for the linearity of proportional hazards for continuous variables using an interaction term between age and the logarithm of age. In the case of significant interaction terms, we used age as a categorical variable using 5-year 
Table 1 Baseline characteristics of patients initiating DPP-4 inhibitors, SGLT2 inhibitors or GLP-1 receptor agonists

\begin{tabular}{|c|c|c|c|c|}
\hline Characteristic & DPP-4i & SGLT2i & GLP-1RA & Total cohort \\
\hline No of patients & $3817(50.7)$ & $2851(37.9)$ & $855(11.4)$ & $7523(100)$ \\
\hline Gender (female) (n, \%)* & $1849(48.4)$ & $1088(38.2)$ & $392(45.8)$ & $3329(44.3)$ \\
\hline Patient age (median, IQR) & $69(61-78)$ & $64(58-70)$ & $62(56-68)$ & $66(59-74)$ \\
\hline \multicolumn{5}{|l|}{ Duration of diabetes therapy* } \\
\hline \multicolumn{5}{|l|}{ Antidiabetic medicines used in the past 135 days } \\
\hline Metformin $^{*}$ & $3009(78.8)$ & $2626(92.1)$ & $790(92.4)$ & $6425(85.4)$ \\
\hline Sulphonylureas* & $3029(79.4)$ & $1970(69.1)$ & $577(67.5)$ & $5576(74.1)$ \\
\hline Other antidiabetic medicines $\dagger^{\star}$ & $134(3.5)$ & $73(2.6)$ & $39(4.6)$ & $246(3.3)$ \\
\hline Three antidiabetic medicine classes & $11(0.3)$ & $16(0.6)$ & $6(0.7)$ & $33(0.4)$ \\
\hline \multicolumn{5}{|l|}{ Use of insulin } \\
\hline Use of insulin therapy in the past year* & $248(6.5)$ & $798(28.0)$ & $333(38.9)$ & $1379(18.3)$ \\
\hline \multicolumn{5}{|l|}{ Previous hospitalisation } \\
\hline Hospital admission due to CV causes in the past year* & $263(6.9)$ & $171(6.0)$ & $42(4.9)$ & $476(6.3)$ \\
\hline Hospital admission due to CV causes (excluding HF) in the past year & $224(5.9)$ & $160(5.6)$ & $38(4.4)$ & $422(5.6)$ \\
\hline Hospital admission due to HF in the past year ${ }^{\star}$ & $44(1.2)$ & $13(0.5)$ & $6(0.7)$ & $63(0.8)$ \\
\hline Hospital admission due to Ml or stroke in the past year & $46(1.2)$ & $40(1.4)$ & $6(0.7)$ & $92(1.2)$ \\
\hline Hospital admission due to type 2 diabetes in the past year* & $59(1.5)$ & $33(1.2)$ & $27(3.2)$ & $119(1.6)$ \\
\hline Hospital admission due to/with cancer in the past 5 years $\S^{*}$ & $198(5.2)$ & $116(4.1)$ & $32(3.7)$ & $346(4.6)$ \\
\hline MRA & $172(4.5)$ & $118(4.1)$ & $41(4.8)$ & $331(4.4)$ \\
\hline Beta blocker* & $1489(39.0)$ & $1181(41.4)$ & $371(43.4)$ & $3041(40.4)$ \\
\hline Calcium channel blocker* & $1423(37.3)$ & $1045(36.7)$ & $353(41.3)$ & 2821 (37.5) \\
\hline ACE-inhibitor or ARBף ${ }^{*}$ & $2773(72.6)$ & 2164 (75.9) & $671(78.5)$ & $5608(74.5)$ \\
\hline Statin $^{*}$ & $2211(57.9)$ & $1865(65.4)$ & $511(59.8)$ & $4587(61.0)$ \\
\hline Other lipid modifying agent* & $183(4.8)$ & $195(6.8)$ & $68(8.0)$ & $446(5.9)$ \\
\hline Antidepressant ${ }^{\star}$ & $487(12.8)$ & $333(11.7)$ & $141(16.5)$ & $961(12.8)$ \\
\hline Anxiolytic, hypnotic, or sedative ${ }^{*}$ & $587(15.4)$ & $351(12.3)$ & $96(11.2)$ & $1034(13.7)$ \\
\hline NSAID* & 761 (19.9) & $657(23.0)$ & $213(24.9)$ & $1631(21.7)$ \\
\hline \multicolumn{5}{|l|}{ Time of cohort entry* } \\
\hline From 30 June 2014 to 29 June 2016 & $2236(58.6)$ & $837(29.4)$ & $345(40.4)$ & $3418(45.4)$ \\
\hline From 30 June 2016 to 30 June 2018 & $1581(41.4)$ & $2014(70.6)$ & $510(59.6)$ & $4105(54.6)$ \\
\hline
\end{tabular}

*Indicates a statistically significant difference ( $p$ value for omnibus test $<0.05$ ) among antidiabetic medicine groups.

†Repaglinide or acarbose.

†Excluding insulin.

$\S$ Cancer as a main or concomitant diagnosis; this covariate was included only in the all-cause death model as cancer was the second most common cause of death in our cohort.

IIncluding combination of valsartan and sacubitril (Eight patients received combination of valsartan and sacubitril within 135 days preceding index date. Due to the small number of patients using a combination of valsartan and sacubitril, this covariate was not separately included in the Cox regression models).

ACE, angiotensin-converting enzyme inhibitors; ARB, angiotensin II receptor blockers; CV, cardiovascular; DPP-4i, dipeptidyl peptidase-4 inhibitor; GLP-1RA, glucagon-like peptide-1 receptor agonist; HF, heart failure; Ml, myocardial infarction; MRA, mineralocorticoid (aldosterone) receptor antagonists; NSAID, nonsteroidal anti-inflammatory drug; SGLT2i, sodium-glucose co-transporter 2 inhibitor. 
groupings. Furthermore, several other interaction terms were introduced into the models to account for the association between antidiabetic medicine group and baseline patient characteristics that statistically significantly differed between groups.

The best model for each outcome was assessed using the statistically significant difference in the result of omnibus tests of different models. Omnibus test results are presented as likelihood ratios of $\chi^{2}$ test with their respective df. Models were deemed to be statistically significantly better than other models if their likelihood ratio increased by at least 3.84 for one df. The proportional evaluated using the PHA test, based on Schoenfeld residuals. In addition, the collinearity among the covariates was assessed by inspecting covariance matrix.

We performed a number of different sensitivity analyses to test result robustness. Specifically, we used an on-treatment approach, whereby patients were followed from the start date until they either discontinued a novel antidiabetic medicine group or initiated a comparator medicine. Patients who discontinued treatment were censored at the date of their last prescription, taking into account a grace period of 135 days after the last prescription. Patients who initiated comparator medicine were censored at the date of the first prescription of comparator medicine. Another sensitivity analysis consisted of fitting a model, where use of insulin was considered to be a time-dependent variable (time-dependent insulin model) in order to capture the effect of initiating insulin after the start date.

$\mathrm{P}$ values smaller than 0.05 were deemed to indicate statistical significance. Data linkage and cohort selection were performed using IBM SPSS Statistics for Windows, V.26.0, and statistical analyses were conducted using Stata Statistical Software: Release 16.

Patient and public involvement statement Patients were not involved in this study. hazards assumption (PHA) of Cox regression models was

RESULTS

\section{Cohort and patient characteristics}

During the period 30 June 2014 to 30 June 2018, we identified 3817 new users of DPP-4i (sitagliptin, 60.4\%; linagliptin, 28.4\%; vildagliptin, $10.7 \%$; saxagliptin, $0.4 \%$; alogliptin, $0.1 \%$ ), 2851 new users of SGLT2i (empagliflozin, 60.3\%; dapagliflozin, 39.7\%) and 855 new users of GLP-1RA (liraglutide, $44.9 \%$; dulaglutide, $32.6 \%$; exenatide, $17.5 \%$; lixisenatide, $4.9 \%)$. The mean (SD) age of the study cohort was $66(10)$ years and $3329(44.3 \%)$ were women. More than $80 \%$ of patients in the study cohort were prescribed antihypertensives and $61 \%$ of patients were prescribed statin. Approximately $6 \%$ of patients had a history of CV events before cohort entry. Baseline characteristics of patients are shown in table 1 .

\section{Intention-to-treat analyses}

There were 437 MACE events, $234 \mathrm{CV}$ deaths, $205 \mathrm{HF}$ events and 617 all-cause deaths during 21365 personyears of follow-up in the ITT analysis (median (IQR) follow-up 2.8 (1.9-3.8) years). Patients from SGLT2i, GLP-1RA and DPP-4i group were followed for a median (IQR) of 2.5 (1.8-3.2) years, 2.5 (1.8-3.8) years and 3.2 (2.1-4.1) years, respectively.

Results from the full covariate model for MACE showed that, when compared with DPP-4i, SGLT2i add-on therapy (dapagliflozin or empagliflozin) was associated with a 1.5 -fold lower risk of MACE ( $\mathrm{HR}=0.67 ; 95 \%$ CI 0.51 to $0.87 ; \mathrm{p}=0.003)$. Comparing the number of events per 1000 person-years in these two cohorts (26.9 vs 12.6, figure 2) would yield to 2.1-fold lower risk of MACE for SGLT2i. However, this is an unbalanced estimate because patients in the SGLT2i group were on average 5 years younger, which per se leads to a lower risk of developing MACE. On the other hand, the model derived estimate (1.5-fold lower risk) was obtained at mean values for the entire cohort of all covariates included in the model. This

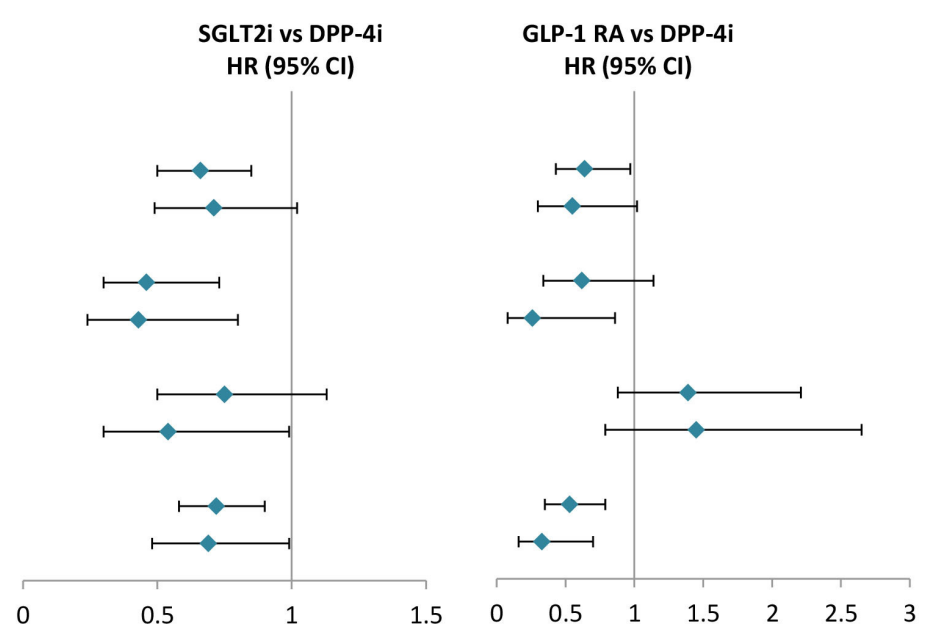

Figure 2 Association between use of SGLT2i and GLP-1RA compared with DPP-4i and the risk of MACE, CVD, HF and allcause death. ACD, all-cause death; CVD, cardiovascular death; DPP-4i, dipeptidyl peptidase-4 inhibitor; GLP-1RA, glucagonlike peptide-1 receptor agonist; HF, heart failure; MACE, major adverse cardiovascular event; ITT, intention-to-treat; SGLT2i, sodium-glucose co-transporter 2 inhibitor. 
Table 2 Final covariate models for all outcomes in intention-to-treat analyses

\begin{tabular}{|c|c|c|c|c|}
\hline \multirow[b]{2}{*}{ Covariates } & MACE & $\begin{array}{l}\text { Cardiovascular } \\
\text { death }\end{array}$ & \multirow{2}{*}{$\begin{array}{l}\text { Heart failure } \\
\text { HR }(95 \% \mathrm{Cl})\end{array}$} & \multirow{2}{*}{$\begin{array}{l}\text { All-cause death } \\
\text { HR }(95 \% \mathrm{Cl})\end{array}$} \\
\hline & HR $(95 \%$ CI) & HR (95\% Cl) & & \\
\hline GLP-1RA vs DPP-4i & $\begin{array}{l}0.64(0.43 \text { to } 0.97) \\
p=0.034\end{array}$ & $\begin{array}{l}0.62(0.34 \text { to } 1.14) \\
p=0.128\end{array}$ & $\begin{array}{l}1.39(0.88 \text { to } 2.21) \\
p=0.157\end{array}$ & $\begin{array}{l}0.53(0.35 \text { to } 0.79) ; \\
\mathrm{p}=0.002\end{array}$ \\
\hline Age & $1.06(1.04 \text { to } 1.07)^{\star}$ & $1.08(1.07 \text { to } 1.10)^{\star}$ & $\mathrm{p}<0.0005 \dagger$ & $1.07(1.06 \text { to } 1.08)^{\star}$ \\
\hline Duration of diabetes therapy $\ddagger$ & $1.46(1.08 \text { to } 1.97)^{\star}$ & 1.44 (0.94 to 2.18$)$ & $1.06(0.71$ to 1.58$)$ & 1.23 (0.97 to 1.58$)$ \\
\hline Use of insulin (yes vs no) & $1.42(1.09 \text { to } 1.85)^{\star}$ & 1.44 (0.996 to 2.08$)$ & 1.08 (0.73 to 1.61$)$ & $1.29(1.02 \text { to } 1.57)^{\star}$ \\
\hline T2D hospitalisation§ (yes vs no) & $2.53(1.60 \text { to } 4.00)^{*}$ & $3.24(1.88 \text { to } 5.56)^{\star}$ & 1.68 (0.76 to 3.72$)$ & $2.57(1.78 \text { to } 3.71)^{\star}$ \\
\hline CV hospitalisation§ (yes vs no) & $1.63(1.23 \text { to } 2.16)^{\star}$ & $1.63(1.14 \text { to } 2.33)^{\star}$ & NA & 1.23 (0.96 to 1.57$)$ \\
\hline Time of cohort entry†† & $p>0.29$ & $0.82(0.60$ to 1.11$)$ & $0.76(0.55$ to 1.05$)$ & $p>0.29$ \\
\hline Sulphonylureas (yes vs no) & $p>0.29$ & $p>0.29$ & $p>0.29$ & 1.24 (0.99 to 1.57$)$ \\
\hline Metformin (yes vs no) & $0.78(0.62 \text { to } 0.97)^{\star}$ & 0.76 (0.57 to 1.01$)$ & $0.71(0.52 \text { to } 0.97)^{*}$ & $p>0.2 \eta$ \\
\hline Anticoagulants (yes vs no) & $p>0.29$ & $1.52(1.07 \text { to } 2.15)^{\star}$ & $2.68(1.88 \text { to } 3.83)^{\star}$ & $1.45(1.16 \text { to } 1.81)^{\star}$ \\
\hline Platelet inhibitors (yes vs no) & $1.20(0.98$ to 1.47$)$ & $1.35(1.01 \text { to } 1.81)^{\star}$ & $1.67(1.21 \text { to } 2.29)^{*}$ & $1.26(1.05 \text { to } 1.51)^{\star}$ \\
\hline Antiarrhythmics $\ddagger \ddagger$ (yes vs no) & 1.25 (0.96 to 1.62$)$ & $p>0.29$ & $1.40(1.01 \text { to } 1.93)^{*}$ & 1.16 (0.93 to 1.43$)$ \\
\hline Loop diuretics (yes vs no) & $1.67(1.31 \text { to } 2.13)^{\star}$ & $2.52(1.87 \text { to } 3.39)^{\star}$ & $3.61(2.61 \text { to } 4.99)^{*}$ & $2.23(1.84 \text { to } 2.70)^{\star}$ \\
\hline Thiazides and other diuretics (yes vs no) & $p>0.2 \rrbracket$ & $p>0.2 \rrbracket$ & $p>0.29$ & $p>0.2 \emptyset$ \\
\hline MRA (yes vs no) & 1.33 (0.94 to 1.89$)$ & $1.56(1.03 \text { to } 2.35)^{\star}$ & $2.15(1.48 \text { to } 3.10)^{*}$ & $1.40(1.07 \text { to } 1.84)^{*}$ \\
\hline Anxiolytic, hypnotic or sedative (yes vs no) & $p>0.29$ & $p>0.29$ & $p>0.2 \rrbracket$ & $1.32(1.08 \text { to } 1.61)^{\star}$ \\
\hline NSAID (yes vs no) & $1.25(0.998 \text { to } 1.58)^{\star}$ & $p>0.2 \uparrow$ & $p>0.2 \rrbracket$ & 1.16 (0.95 to 1.42$)$ \\
\hline Result of omnibus test (df) & $415.36(17 \mathrm{df})$ & 461.81 (16 df) & 486.88 (21 df) & 867.68 (21 df) \\
\hline
\end{tabular}

*Indicates statistical significance ( $p$ value $<0.05)$.

†Age was treated as a categorical variable, age group 65-69 served as a reference category, $<55$ versus $65-69(\mathrm{HR}=0.15 ; 95 \% \mathrm{Cl} 0.04$ to 0.63$)$; $55-64$ versus $65-69$ ( $\mathrm{HR}=0.55 ; 95 \% \mathrm{Cl} 0.32$ to 0.92$)$; $\geq 70$ versus $65-69$ ( $\mathrm{HR}=1.51 ; 95 \% \mathrm{Cl} 0.998$ to 2.28 ).

$\ddagger$ Patients with diabetes therapy duration of 5 years or more were compared with patients with diabetes therapy duration of less than 5 years. $\S$ Hospital admission in the past year.

IRemoval probability for stepwise backward selection based on Wald test was set to 0.2 for all analyses, therefore, covariates with $p$ values of 0.2 or greater were excluded from the model and their respective HRs were not calculated.

${ }^{*}$ Cancer hospitalisation (main or concomitant diagnosis) in the past 5 years.

††From 30 June 2016 to 30 June 2018 versus from 30 June 2014 to 29 June 2016.

¥¥Including antiarrhythmics, class I and III, cardiac glycosides, vasodilators.

ACE, angiotensin-converting enzyme inhibitors; ARB, angiotensin II receptor blockers; CV, cardiovascular; DPP-4i, dipeptidyl peptidase-4 inhibitor; GLP-1RA, glucagon-like peptide-1 receptor agonist; HF, heart failure; MACE, major adverse cardiovascular event; MRA, mineralocorticoid (aldosterone) receptor antagonists; NA, not applicable; NSAID, non-steroidal anti-inflammatory drug; SGLT2i, sodium-glucose co-transporter 2 inhibitor; T2D, type 2 diabetes.

means that differences in the baseline covariates among the groups are taken into account. Similar results were obtained for GLP-1RA add-on therapy (HR $=0.65 ; 95 \%$ CI 0.43 to $0.99 ; \mathrm{p}=0.043$ ). The relative effects among the antidiabetic medicine groups on MACE in the final covariate
ITT model were similar to those in the full covariate model (table 2, figure 2).

Compared with DPP-4i add-on therapy, SGLT2i therapy was associated with a 2.2-fold lower risk of $\mathrm{CV}$ death $(\mathrm{HR}=0.46 ; 95 \% \mathrm{CI} 0.30$ to $0.73 ; \mathrm{p}=0.001)$. In contrast, 

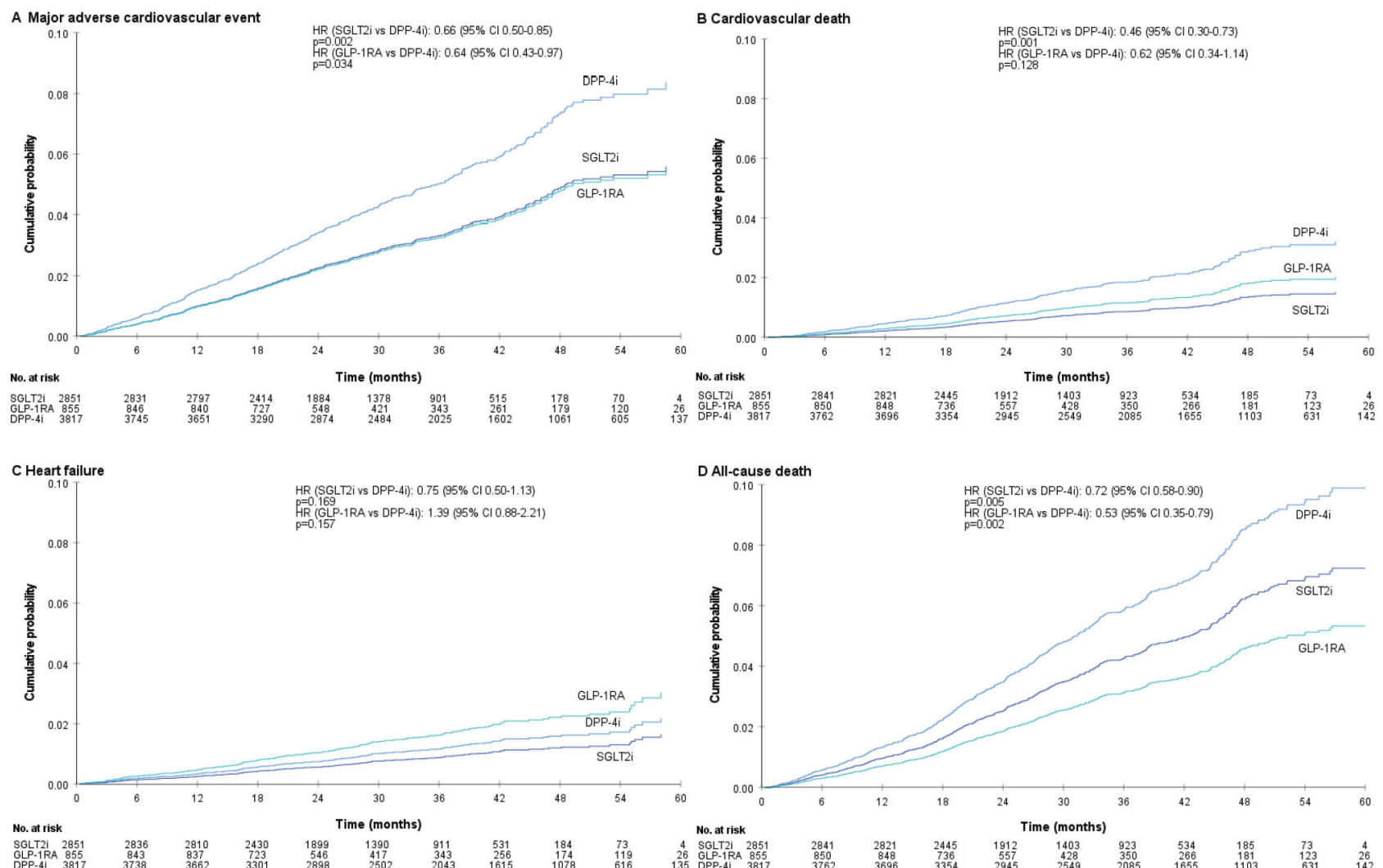

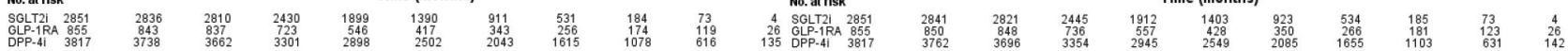

Figure 3 Cumulative probability of (A) major adverse cardiovascular events, (B) cardiovascular death, (C) heart failure and (D) all-cause death in patients initiating DPP-4 inhibitors, GLP-1 receptor agonists or SGLT2 inhibitors at mean values of all included covariates for intention-to-treat analyses. DPP-4i, dipeptidyl peptidase-4 inhibitor; GLP-1RA, glucagon-like peptide-1 receptor agonist; SGLT2i, sodium-glucose co-transporter 2 inhibitor.

when GLP-1RA and DPP-4i were compared, there was a non-significant, although marked, reduction in the risk of $\mathrm{CV}$ death (HR=0.62; $95 \%$ CI 0.34 to $1.14 ; \mathrm{p}=0.128)$ in the GLP-1RA group. We also assessed the risk of all-cause death. The majority of patients died from CV causes $(38 \%$ of all deaths), followed by cancer (33\% of all deaths) and complications due to T2D ( $8 \%$ of all deaths). Results from the model of all-cause deaths confirmed that patients who used either SGLT2i or GLP-1RA did not die more frequently from non-CV causes compared with those who used DPP-4i. In the ITT analysis, both SGLT2i and GLP1RA add-on therapy were associated with a lower risk of all-cause death (table 2, figures 2 and 3). In addition, preliminary results of the ITT analysis suggest that GLP1RA use is associated with a lower risk of non-diabetes and non-CV mortality compared with DPP-4i use (covariate adjusted $\mathrm{HR}=0.36 ; 95 \% \mathrm{CI} 0.19$ to $0.70 ; \mathrm{p}=0.002$ ), while there is no difference in risk of non-diabetes and non-CV mortality between SGLT2i and DPP-4i groups (covariate adjusted $\mathrm{HR}=0.89 ; 95 \%$ CI 0.68 to $1.18 ; \mathrm{p}=0.432$ ).

A non-significantly lower risk of HF hospitalisation was observed in patients initiating SGLT2i add-on therapy (HR $=0.75 ; 95 \%$ CI 0.50 to $1.13 ; \mathrm{p}=0.169)$ compared with patients initiating DPP-4i add-on therapy. In contrast, when comparing the GLP-1RA and DPP-4i groups, there was a non-significantly increased risk of $\mathrm{HF}(\mathrm{HR}=1.39$;
95\% CI 0.88 to $2.21 ; \mathrm{p}=0.157)$ in the GLP-1RA group (table 2, figures 2 and 3 ).

There were no statistically significant interaction terms between antidiabetic medicine group and baseline patient characteristics in any of the models, suggesting that the relative effect among antidiabetic medicine groups on study outcomes was not different due to the variations in patient characteristics between antidiabetic medicine groups (eg, age, gender, prior use of metformin or sulphonylureas and others). Detailed results of the full covariate model for MACE are presented in online supplemental table S3. Detailed results of final covariate models for ITT analyses are presented in table 2. Figure 2 shows forest plot for all outcomes for ITT and on-treatment analyses. Figure 3 shows the cumulative probability of each outcome at mean values of all included covariates separately for antidiabetic medicine group.

\section{Sensitivity analyses}

On-treatment analyses

There were 206 MACE events, $101 \mathrm{CV}$ deaths, $101 \mathrm{HF}$ events and 232 all-cause deaths during 11251 person-years of follow-up in the on-treatment analysis. Patients from SGLT2i, GLP-1RA and DPP-4i group were followed for a median (IQR) of $1.4(0.6-2.2)$ years, $1.4(0.7-2.2)$ years and $1.2(0.5-2.2)$ years, respectively. The point estimates 
of the HRs for all outcomes in the on-treatment analysis were in line with the ITT findings (figure 2, online supplemental table S4 and figure S1). However, while in the ITT analysis, the effect of SGLT2i compared with DPP-4i on HF hospitalisation risk was not significant, the on-treatment analysis revealed a 1.9-fold lower risk of HF hospitalisation in patients initiating SGLT2i (HR $=0.54 ; 95 \% \mathrm{CI}$ 0.30 to $0.99 ; \mathrm{p}=0.047$ ). Point estimates of HRs for CV and all-cause death for the GLP-1RA group were lower than in the ITT analysis and less accurate because of the smaller number of events in the GLP-1RA group. Detailed results of final covariate models for on-treatment analyses are presented in online supplemental table S4.

\section{Time-dependent insulin models}

The effects of the SGLT2i and GLP-1RA groups on risk of all outcomes in the time-dependent insulin models were similar to the ITT findings. The effects of insulin in the time-dependent insulin models were also similar to those in the ITT analysis. The effect of insulin was statistically significant in the time-dependent insulin models for MACE (HR=1.25; 95\% CI 1.02 to $1.54 ; \mathrm{p}=0.034)$ and all-cause death ( $\mathrm{HR}=1.61 ; 95 \%$ CI 1.36 to $1.91 ; \mathrm{p}<0.0005)$. This model revealed the following numbers of patients using insulin at the time of the event or at the end of follow-up: (1) 1100 (29\%) patients from the DPP-4i group, (2) 1124 (39\%) patients from the SGLT2i group and (3) 475 (56\%) patients from the GLP-1RA group.

\section{DISCUSSION}

This is the first real-world observational study to compare $\mathrm{CV}$ outcomes of patients being treated with different novel antidiabetic medicine groups in one study. Importantly, this study evaluated the use of novel antidiabetic medicines as add-on therapy to an existing background therapy, and it had a long follow-up period of 2.8 years. The study showed that SGLT2i add-on therapy was associated with a 1.5-fold lower risk of MACE and a 2.2-fold lower risk of $\mathrm{CV}$ death compared with DPP-4i add-on therapy in patients with T2D. In addition, SGLT2i were associated with a 1.9-fold lower risk of hospitalisation due to HF in the on-treatment analysis, while ITT analysis revealed a non-significant reduction in risk of HF hospitalisation. GLP-1RA add-on therapy was associated with a 1.6-fold lower risk of MACE, a non-significantly lower risk of $\mathrm{CV}$ death, and a non-significantly higher risk of $\mathrm{HF}$ hospitalisation compared with DPP-4i.

In comparison with other studies comparing SGLT2i and DPP-4i ${ }^{15}{ }^{16}$ the current study revealed a greater risk reduction of MACE and CV death in patients treated with SGLT2i. The greater risk reduction of MACE and CV death found here is likely due to the longer follow-up period of this study. Similar to SGLT2i, GLP-1RA treatment was also found to result in a greater risk reduction of MACE compared with other studies that used DPP-4i as a comparator. ${ }^{17}{ }^{21}$ Moreover, a non-significant risk reduction of CV death was found, which was of greater magnitude to that found by Svanström et al: ${ }^{17}$ the nonsignificance of our result is likely due to the small number of patients in the GLP-1RA group and the small absolute number of events in this group, which led to wide CIs.

Current study also revealed a risk reduction of allcause death in patients treated with SGLT2i or GLP-1RA compared with those treated with DPP-4i. While the risk reduction of all-cause death in the SGLT2i group was mostly due to the risk reduction of CV death, an additional risk reduction in all-cause death was observed for GLP1RA, which might be due to non-CV mortality. Indeed, the preliminary results of our study suggest that GLP1RA use is associated with a lower risk of non-CV death compared with DPP-4i use, whereas there is no difference between SGLT2i and DPP-4i groups. However, evaluating the relative effect among antidiabetic medicine groups on non-CV mortality was not the aim of the current study. A new study including a new cohort of patients is needed to investigate this effect. However, findings of the current study are in contrast to the findings of a recent network meta-analysis ${ }^{26}$ that found no additional benefit of GLP1RA on mortality beyond the reduction of CV mortality risk. A higher risk reduction in non-CV mortality in users of GLP-1RA in the current study might be due to death from cancer. Recent meta-analyses showed no association between the risk of different types of cancer and the use of GLP-1RA ${ }^{27}$ or DPP-4i. ${ }^{28}$ However, further studies are needed to investigate the possible beneficial effect of GLP-1RA on cancer mortality.

The magnitude of reduction in HF risk in the on-treatment analysis was similar to other studies that compared SGLT2i and DPP-4i. ${ }^{9} 12151618$ In contrast, the ITT analysis did not show statistically significant reduction in HF risk in patients treated with SGLT2i. According to previous studies, the effect of SGLT2i on the risk reduction of HF could be observed soon after therapy initiation. ${ }^{18} \mathrm{~A}$ longer follow-up period in our study, during which many patients switched therapy, might possibly explain why statistical significance in the ITT analysis was not reached between the SGLT2i and DPP-4i groups. A noticeable number (13\%) of patients in the DPP-4i group switched to SGLT2i during follow-up, which may have resulted in a smaller difference in HF risk between the two groups.

There is not yet clear evidence of any different effect on HF risk of GLP-1RA compared with DPP-4i. Our study showed that GLP-1RA add-on therapy was associated with a 1.4-fold higher risk of HF hospitalisation compared with DPP-4i add-on therapy. These results are in agreement with the study by Dawwas et al which showed that patients treated with DPP-4i had a 1.2-fold lower risk of HF compared with those treated with GLP-1RA. ${ }^{13}$ In contrast, two other observational studies comparing initiators of GLP-1RA and DPP-4i showed no difference in the risk of HF hospitalisation between DPP-4i and GLP-1RA groups. ${ }^{1721}$

In addition, in our study, the use of statins was associated with a lower risk of $\mathrm{CV}$ events and lower mortality rates. We assume that patients treated with statins had 
lower low-density lipoprotein (LDL) cholesterol levels than patients not treated with statins, resulting in the lower incidence of CV events. ${ }^{29}$ However, to confirm this assumption, data on LDL cholesterol levels for the entire cohort would be needed.

\section{Strengths}

Potential immortal time bias was avoided by using incident user design and comparing $\mathrm{CV}$ outcomes of patients taking antidiabetic medicines that are used at similar disease stages. In addition, as cohort entry was defined by at least two prescriptions, both issued within a 135-day period, of a novel antidiabetic medicine group, follow-up started 135 days after the first prescription. ${ }^{30}$ Furthermore, a lag period after the first prescription was used to reduce a potential protopathic bias. ${ }^{31}$ Moreover, we developed a Cox regression model that included all available confounding variables that might affect the association between antidiabetic medicine groups and outcome of interest. ${ }^{25}$ We did not use propensity score matching for three treatment groups because in this case many patients (about $80 \%$ ) would have been excluded from the final study sample, leading to less generalisable results. ${ }^{32}$ Furthermore, the number of events in our models was at least eight times higher than the number of confounders included in the models. In such a case, the use of regression models instead of propensity scores leads to more accurate and less biased estimates. ${ }^{33}$ In addition, the relatively long follow-up period in this study enabled us to detect differences in $\mathrm{CV}$ events and deaths that may not be apparent at the shorter follow-up durations of previous studies. Moreover, on-treatment analyses and sensitivity analyses confirmed that the results of our primary analyses were robust.

\section{Limitations}

The study results must be interpreted in the context of the following study limitations. First, data regarding other comorbidities and date of diabetes diagnosis was not included in healthcare databases used in this study. We addressed this limitation by using data on all dispensed outpatient prescriptions as a proxy for comorbidities. We used time from the first dispensed prescription for antidiabetic medicine as a proxy for duration of diabetes therapy. However, because data before 2009 were not available, we could only distinguish between patients who had suffered from diabetes for more or less than 5 years. Second, we could not adjust for some potential confounders since we did not have data on laboratory values of glycated haemoglobin (HbAlc), LDL cholesterol, blood pressure, left ventricular function, smoking status or body mass index. However, we indirectly adjusted for some of these confounders by using data on dispensed outpatient medicines. Third, inclusion period started at the date of introduction of the first medicine from the SGLT2i group (dapagliflozin) in the Slovenian market. Results from the EMPA-REG study became available soon after the introduction of SGLT2i medicines in the Slovenian market, and this may have encouraged prescribing of SGLT2i (in particular, empagliflozin) to patients with higher CV risk; this possibly resulted in a protopathic bias. To minimise potential protopathic bias, we used a lag period after first prescription.

\section{CONCLUSIONS}

This real-world study revealed that SGLT2i and GLP-1RA as add-on therapy improve CV morbidity and mortality in patients with T2D compared with DPP-4i as add-on therapy. SGLT2i and GLP-1RA add-on therapy was associated with a lower risk of MACE and CV death compared with DPP-4i add-on therapy. Furthermore, SGLT2i were associated with a lower risk of HF, while GLP-1RA use was associated with a non-significantly higher risk of HF compared with DPP-4i. The results of this study may help to inform treatment decisions when selecting an optimal add-on antidiabetic medicine in order to reduce CV morbidity and mortality in patients with T2D.

Contributors All authors (SZ, MK and IL) were involved in the study design. SZ analysed the data and all authors interpreted the data. SZ prepared a draft of manuscript, which was critically revised and upgraded by MK and IL. All authors read and approved the final version of the manuscript.

Funding This work was supported by the Slovenian Research Agency (Programme Group No. P1-0189). The Slovenian Research Agency had no role in the design and conduct of the study; analysis and interpretation of data; preparation, approval and submission of the manuscript for publication.

Competing interests None declared.

Patient consent for publication Not required.

Ethics approval The study was approved by the National Medical Ethics Committee (registration number: 0120-264/2019/5).

Provenance and peer review Not commissioned; externally peer reviewed.

Data availability statement Data may be obtained from a third party and are not publicly available. Data were obtained from a third party (National Institute of Public Health - Slovenia, www.nijz.si/en) and are not publicly available.

Supplemental material This content has been supplied by the author(s). It has not been vetted by BMJ Publishing Group Limited (BMJ) and may not have been peer-reviewed. Any opinions or recommendations discussed are solely those of the author(s) and are not endorsed by BMJ. BMJ disclaims all liability and responsibility arising from any reliance placed on the content. Where the content includes any translated material, BMJ does not warrant the accuracy and reliability of the translations (including but not limited to local regulations, clinical guidelines, terminology, drug names and drug dosages), and is not responsible for any error and/or omissions arising from translation and adaptation or otherwise.

Open access This is an open access article distributed in accordance with the Creative Commons Attribution Non Commercial (CC BY-NC 4.0) license, which permits others to distribute, remix, adapt, build upon this work non-commercially, and license their derivative works on different terms, provided the original work is properly cited, appropriate credit is given, any changes made indicated, and the use is non-commercial. See: http://creativecommons.org/licenses/by-nc/4.0/.

\section{ORCID iDs}

Spela Zerovnik http://orcid.org/0000-0003-3918-0929

Mitja Kos http://orcid.org/0000-0002-6801-6450

Igor Locatelli http://orcid.org/0000-0002-0052-8986

\section{REFERENCES}

1 Zinman B, Wanner C, Lachin JM, et al. Empagliflozin, cardiovascular outcomes, and mortality in type 2 diabetes. $N$ Engl J Med 2015;373:2117-28. 
2 Neal B, Perkovic V, Mahaffey KW, et al. Canagliflozin and cardiovascular and renal events in type 2 diabetes. $N$ Engl $\mathrm{J}$ Med 2017;377:644-57.

3 Wiviott SD, Raz I, Bonaca MP, et al. Dapagliflozin and cardiovascular outcomes in type 2 diabetes. N Engl J Med 2019;380:347-57.

4 Marso SP, Bain SC, Consoli A, et al. Semaglutide and cardiovascular outcomes in patients with type 2 diabetes. N Engl J Med 2016;375:1834-44.

5 Marso SP, Daniels GH, Brown-Frandsen K, et al. Liraglutide and cardiovascular outcomes in type 2 diabetes. $N$ Engl J Med 2016;375:311-22.

6 Holman RR, Bethel MA, Mentz RJ, et al. Effects of once-weekly exenatide on cardiovascular outcomes in type 2 diabetes. $N$ Engl $J$ Med 2017;377:1228-39.

7 Pfeffer MA, Claggett B, Diaz R, et al. Lixisenatide in patients with type 2 diabetes and acute coronary syndrome. N Engl J Med 2015;373:2247-57.

8 Gerstein HC, Colhoun HM, Dagenais GR, et al. Dulaglutide and cardiovascular outcomes in type 2 diabetes (REWIND): a doubleblind, randomised placebo-controlled trial. Lancet 2019;394:121-30.

9 Patorno E, Goldfine AB, Schneeweiss S, et al. Cardiovascular outcomes associated with canagliflozin versus other nongliflozin antidiabetic drugs: population based cohort study. BMJ 2018;360:k119.

10 Patorno E, Pawar A, Franklin JM, et al. Empagliflozin and the risk of heart failure hospitalization in routine clinical care. Circulation 2019;139:2822-30.

11 Ryan PB, Buse JB, Schuemie MJ, et al. Comparative effectiveness of canagliflozin, SGLT2 inhibitors and non-SGLT2 inhibitors on the risk of hospitalization for heart failure and amputation in patients with type 2 diabetes mellitus: a real-world meta-analysis of 4 observational databases (OBSERVE-4D). Diabetes Obes Metab 2018;20:2585-97.

12 Dawwas GK, Smith SM, Park H. Cardiovascular outcomes of sodium glucose cotransporter-2 inhibitors in patients with type 2 diabetes. Diabetes Obes Metab 2019;21:28-36.

13 Dawwas GK, Smith SM, Park H. Risk of heart failure hospitalization among users of dipeptidyl peptidase-4 inhibitors compared to glucagon-like peptide-1 receptor agonists. Cardiovasc Diabetol 2018;17:102.

14 Birkeland KI, Jørgensen ME, Carstensen B, et al. Cardiovascular mortality and morbidity in patients with type 2 diabetes following initiation of sodium-glucose co-transporter-2 inhibitors versus other glucose-lowering drugs (CVD-REAL Nordic): a multinational observational analysis. Lancet Diabetes Endocrinol 2017;5:709-17.

15 Pasternak B, Ueda P, Eliasson B, et al. Use of sodium glucose cotransporter 2 inhibitors and risk of major cardiovascular events and heart failure: Scandinavian register based cohort study. BMJ 2019;366:14772.

16 Persson F, Nyström T, Jørgensen ME, et al. Dapagliflozin is associated with lower risk of cardiovascular events and all-cause mortality in people with type 2 diabetes (CVD-REAL Nordic) when compared with dipeptidyl peptidase-4 inhibitor therapy: a multinational observational study. Diabetes Obes Metab 2018;20:344-51.

17 Svanström H, Ueda $\mathrm{P}$, Melbye $\mathrm{M}$, et al. Use of liraglutide and risk of major cardiovascular events: a register-based cohort study in Denmark and Sweden. Lancet Diabetes Endocrinol 2019;7:106-14.

18 Kim Y-G, Han SJ, Kim DJ, et al. Association between sodium glucose co-transporter 2 inhibitors and a reduced risk of heart failure in patients with type 2 diabetes mellitus: a real-world nationwide population-based cohort study. Cardiovasc Diabetol 2018;17:91.

19 Kosiborod M, Cavender MA, Fu AZ, et al. Lower risk of heart failure and death in patients initiated on sodium-glucose cotransporter-2 inhibitors versus other glucose-lowering drugs: the CVD-REAL study (comparative effectiveness of cardiovascular outcomes in new users of sodium-glucose cotransporter-2 inhibitors). Circulation 2017;136:249-59.

20 Longato E, Di Camillo B, Sparacino G, et al. Cardiovascular outcomes of type 2 diabetic patients treated with SGLT-2 inhibitors versus GLP-1 receptor agonists in real-life. BMJ Open Diabetes Res Care 2020;8:e001451.

21 Longato E, Di Camillo B, Sparacino G, et al. Better cardiovascular outcomes of type 2 diabetic patients treated with GLP-1 receptor agonists versus DPP-4 inhibitors in clinical practice. Cardiovasc Diabetol 2020;19:74

22 Suissa S. Lower risk of death with SGLT2 inhibitors in observational studies: real or bias? Diabetes Care 2018;41:6-10.

23 Davies MJ, D'Alessio DA, Fradkin J, et al. Management of hyperglycemia in type 2 diabetes, 2018. A consensus report by the American diabetes association (ADA) and the European association for the study of diabetes (EASD). Diabetes Care 2018;41:2669-701.

24 Norhammar A, Bodegård J, Nyström T, et al. Dapagliflozin and cardiovascular mortality and disease outcomes in a population with type 2 diabetes similar to that of the DECLARE-TIMI 58 trial: a nationwide observational study. Diabetes Obes Metab 2019;21:1136-45.

25 Biondi-Zoccai G, Romagnoli E, Agostoni P, et al. Are propensity scores really superior to standard multivariable analysis? Contemp Clin Trials 2011;32:731-40.

26 Zheng SL, Roddick AJ, Aghar-Jaffar R, et al. Association between use of sodium-glucose cotransporter 2 inhibitors, glucagon-like peptide 1 agonists, and dipeptidyl peptidase 4 inhibitors with allcause mortality in patients with type 2 diabetes: a systematic review and meta-analysis. JAMA 2018;319:1580-91.

27 Cao C, Yang S, Zhou Z. GLP-1 receptor agonists and risk of cancer in type 2 diabetes: an updated meta-analysis of randomized controlled trials. Endocrine 2019;66:157-65.

28 Dicembrini I, Nreu B, Montereggi C, et al. Risk of cancer in patients treated with dipeptidyl peptidase-4 inhibitors: an extensive meta-analysis of randomized controlled trials. Acta Diabeto 2020;57:689-96.

29 Cholesterol Treatment Trialists' (CTT) Collaborators, Kearney PM, Blackwell L, et al. Efficacy of cholesterol-lowering therapy in 18,686 people with diabetes in 14 randomised trials of statins: a metaanalysis. Lancet 2008;371:117-25.

30 Patorno E, Patrick AR, Garry EM, et al. Observational studies of the association between glucose-lowering medications and cardiovascular outcomes: addressing methodological limitations. Diabetologia 2014;57:2237-50.

31 Tamim H, Monfared AAT, LeLorier J. Application of lag-time into exposure definitions to control for protopathic bias. Pharmacoepidemiol Drug Saf 2007;16:250-8.

32 Deb S, Austin PC, Tu JV, et al. A review of propensity-score methods and their use in cardiovascular research. Can J Cardiol 2016;32:259-65.

33 Cepeda MS, Boston R, Farrar JT, et al. Comparison of logistic regression versus propensity score when the number of events is low and there are multiple confounders. Am J Epidemiol 2003;158:280-7. 